Proyecciones Journal of Mathematics

Vol. 38, No 2, pp. 325-351, June 2019.

Universidad Católica del Norte

Antofagasta - Chile

\title{
Nonlinear elliptic equations in dimension two with potentials which can vanish at infinity
}

\author{
Yony Raúl Santaria Leuyacc \\ Universidade de São Paulo, Brasil \\ Received : October 2017. Accepted : March 2019
}

\begin{abstract}
We will focus on the existence of nontrivial solutions to the following nonlinear elliptic equation

$$
-\Delta u+V(x) u=f(u), \quad x \in \mathbf{R}^{2},
$$

where $V$ is a nonnegative function which can vanish at infinity or be unbounded from above, and $f$ have exponential growth range. The proof involves a truncation argument combined with Mountain Pass Theorem and a Trudinger-Moser type inequality.
\end{abstract}

Keywords: Nonlinear elliptic equations, vanishing potentials, TrudingerMoser inequality.

2010 AMS Subject Classification: 35J60, 35J20,35J15. 


\section{Introduction}

The main purpose of this work is to study the existence of solutions for the following nonlinear elliptic equation:

$$
-\Delta u+V(x) u=f(x, u), \quad x \in \Omega,
$$

where $\Omega \subset \mathbf{R}^{2}, V$ is a continuous potential and the nonlinearity $f$ possesses maximal growth range. It is interesting to compare the equation (1.1) with the case where $\Omega$ is a subset of $\mathbf{R}^{N}, N \geq 3$. In this case, the classical Sobolev theorem asserts that the following embedding is continuous: $H_{0}^{1}(\Omega) \subset L^{q}(\Omega)$ for all $1 \leq q \leq 2^{*}=2 N /(N-2)$. Thus, using variational methods, the maximal growth of the function $f$ is of type: $f(s) \sim|s|^{2^{*}-1}$.

In dimension $N=2$ one has $H_{0}^{1}(\Omega) \subset L^{q}(\Omega)$ for all $q \geq 1$ and $H_{0}^{1}(\Omega) \not \subset$ $L^{\infty}(\Omega)$. In this situation another kind of maximal growth were established independently by Trudinger [20] and Pohožaev [16]. The authors proved that the maximal growth allow us to consider is of type:

$f(s) \sim e^{|s|^{2}}$. Motivated by this result, it was obtained the following characterization of growth: we say that a function $f$ possesses critical exponential growth, if there exists $\alpha_{0}>0$ such that

$$
\lim _{|s| \rightarrow \infty} \frac{f(s)}{e^{\alpha|s|^{2}}}= \begin{cases}0, & \alpha>\alpha_{0}, \\ +\infty, & \alpha<\alpha_{0} .\end{cases}
$$

The equation (1.1) where $f$ possesses critical exponential growth had been studying in many works (see $[5,4,10,11,9,12,3,8,7]$ ).

Adimurthi and Yadava [2], Adimurthi et al. [1] and de Figueiredo et al. [5] studied the problem (1.1), where $\Omega$ is a bounded smooth domain and the potential $V$ is identically zero.

In [9], do Ó and Ruf studied the equation

$$
-\Delta u+V(x) u=f(u), \quad x \in \mathbf{R}^{2},
$$

where $V$ belongs to $\mathcal{C}\left(\mathbf{R}^{2}, \mathbf{R}\right)$ and is a 1-periodic function in $x_{1}$ and $x_{2}$, and 0 is in a spectral gap of the operator $-\Delta+V$.

In [6], it was found a nontrivial solution of the problem

$$
-\Delta u+V(x) u=f(x, u), \quad x \in \mathbf{R}^{2},
$$


where $V: \mathbf{R}^{2} \rightarrow \mathbf{R}$ is a continuous function satisfying

$$
V(x) \geq V_{0}>0, \quad \text { for all } x \in \mathbf{R}^{2},
$$

and the potential $V$ is asymptotically periodic at infinity, that is there exists a continuous 1-periodic function $V_{1}: \mathbf{R}^{2} \rightarrow \mathbf{R}$ such that

(i) $V_{1}(x) \geq V(x)>0$, for all $x \in \mathbf{R}^{2}$.

(ii) $V(x) \rightarrow V_{1}(x)$, as $|x| \rightarrow \infty$.

In [10], it was considered the equation

$$
-\Delta u+V(x) u=f(u)+h(x), \quad x \in \mathbf{R}^{2},
$$

where the potential $V$ satisfy

(i) The function $V(x) \geq V_{0}>0$, for all $x \in \mathbf{R}^{2}$

(ii) $V(x) \rightarrow+\infty$, as $|x| \rightarrow \infty$.

Some extensions of (1.5) can be found in [13, 11, 8].

In [12], the authors studied the existence of nontrivial solutions for the following class of equations

$$
-\epsilon^{2} \Delta u+V(x) u=f(u), \quad x \in \mathbf{R}^{2} .
$$

where $\epsilon$ is a small positive parameter and the potential $V: \mathbf{R}^{2} \rightarrow \mathbf{R}$ satisfies the following conditions:

(i) $V$ is locally Hölder continuous in $\mathbf{R}^{2}$ and there exists a positive constant $V_{0}$ such that

$$
V(x) \geq V_{0}, \quad \text { for all } \quad x \in \mathbf{R}^{2} .
$$

(ii) There exists a bounded domain $\Omega \subset \mathbf{R}^{2}$ such that

$$
\inf _{\Omega} V(x)<\min _{\partial \Omega} V(x) .
$$

Motivated by the above mentioned results, we are interested in studying the equation

$$
-\Delta u+V(x) u=f(u), \quad x \in \mathbf{R}^{2},
$$

where the nonlinearity $f$ possesses critical exponential growth and the potential $V$ can be vanish at infinity. More specifically $V$ satisfies the following assumptions: 
$\left(V_{1}\right) \quad V \in \mathcal{C}\left(\mathbf{R}^{2}, \mathbf{R}\right)$ is a radially symmetric nonnegative function.

$\left(V_{2}\right)$ There exist constants $a, b, R_{0}, L_{a}$ and $L_{b}$, with $0<a<2, b \leq a$, $R_{0} \geq 1, L_{a} \geq R_{0}^{a}$ and $L_{a} R_{0}^{b-a} \leq L_{b}$ such that

$$
\frac{L_{a}}{|x|^{a}} \leq V(x) \leq \frac{L_{b}}{|x|^{b}}, \quad \text { for all } \quad|x| \geq R_{0} .
$$

Before starting the assumptions on the nonlinearity $f$, we define the energy space which will be use to set the variational structure. Following [18], $H_{V, r a d}^{1}\left(\mathbf{R}^{2}\right)$ denote the subspace of the radially symmetric functions in the closure of $\mathcal{C}_{0}^{\infty}\left(\mathbf{R}^{2}\right)$ with respect to the norm

$$
\|u\|=\|u\|_{H_{V}^{1}}:=\left(\int_{\mathbf{R}^{2}}|\nabla u|^{2}+V(x) u^{2} d x\right)^{1 / 2} .
$$

For $1 \leq p<+\infty$, we consider

$$
L_{V, r a d}^{p}\left(\mathbf{R}^{2}\right):=\left\{u \in \mathcal{M}\left(\mathbf{R}^{2}, \mathbf{R}\right): u \text { is radial and } \int_{\mathbf{R}^{2}} V(x)|u|^{p} d x<+\infty\right\},
$$

endowed with the norm

$$
\|u\|_{L_{V}^{p}}=\left(\int_{\mathbf{R}^{2}} V(x)|u|^{p} d x\right)^{1 / p} .
$$

Thus,

$$
H_{V, r a d}^{1}\left(\mathbf{R}^{2}\right)=\left\{u \in L_{V, r a d}^{2}\left(\mathbf{R}^{2}\right):|\nabla u| \in L^{2}\left(\mathbf{R}^{2}\right)\right\} .
$$

We note that $H_{V, r a d}^{1}\left(\mathbf{R}^{2}\right)$ is a Hilbert space endowed with inner product

$$
\langle u, v\rangle:=\int_{\mathbf{R}^{2}}(\nabla u \nabla v+V(x) u v) d x, \quad u, v \in H_{V, r a d}^{1}\left(\mathbf{R}^{2}\right) .
$$

Throughout this paper, we denote by $E$ the space $H_{V, r a d}^{1}\left(\mathbf{R}^{2}\right)$ and by $E^{-1}$ the dual space of $E$ with the usual norm.

Now, we state a basic embedding result (see [18, 19], for a proof).

Lemma 1.1. Suppose $V$ satisfies $\left(V_{1}\right)-\left(V_{2}\right)$. Taking $R_{0}, a$ and $b$ given by $\left(V_{2}\right)$, consider $a^{*}=(4+2 a) /(2-a)$ and $b^{*}=2(2-2 b+a) /(2-a)$. Then,

(i) The embedding $H_{V, \text { rad }}^{1}\left(\mathbf{R}^{2}\right) \hookrightarrow L^{p}\left(\mathbf{R}^{2}\right)$ is continuous for $a^{*} \leq p<+\infty$ and compact for $a^{*}<p<+\infty$. 
(ii) The embedding $H_{V, r a d}^{1}\left(\mathbf{R}^{2}\right) \hookrightarrow L_{V, r a d}^{p}\left(\mathbf{R}^{2}\right)$ is continuous for $b^{*} \leq p<$ $+\infty$ and compact for $b^{*}<p<+\infty$.

(iii) The embedding $H_{V, \text { rad }}^{1}\left(B_{R}\right) \hookrightarrow H^{1}\left(B_{R}\right)$ is continuous for $R>R_{0}$.

Remark 1.2. 1. As a consequence of (iii) and Sobolev embedding theorem, the space $H_{V, r a d}^{1}\left(\mathbf{R}^{2}\right)$ is compactly immersed in $L^{p}\left(B_{R}\right)$ for all $1 \leq p<+\infty$.

We assume the following assumptions on the nonlinearity $f$ :

$\left(H_{1}\right) \quad f \in \mathcal{C}(\mathbf{R})$ and $f(s)=0$ for all $s \leq 0$.

Taking $b^{*} \in \mathbf{R}$ as in Lemma 1.1, consider

$\left(H_{2}\right)$ There exists a constant $\mu>b^{*}$ such that

$$
0<\mu F(s) \leq s f(s), \quad \text { for all } s>0,
$$

where $F(s)=\int_{0}^{s} f(t) d t$.

$\left(H_{3}\right)$ There exist constants $s_{1}>0$ and $M>0$ such that

$$
0<F(s) \leq M f(s), \text { for all } s>s_{1} .
$$

Setting $\mu$ given by $\left(H_{2}\right)$ and $a$ given by $\left(V_{2}\right)$, we suppose:

$\left(H_{4}\right)$ There exists $\theta \geq 4 a /(2-a)$ such that $f(s)=O\left(s^{\mu-1+\theta}\right)$ as $s \rightarrow 0^{+}$.

$\left(H_{5}\right)$ There exists $\alpha_{0}>0$ such that

$$
\lim _{s \rightarrow+\infty} \frac{f(s)}{e^{\alpha|s|^{2}}}= \begin{cases}0, & \alpha>\alpha_{0}, \\ +\infty, & \alpha<\alpha_{0} .\end{cases}
$$

$\left(H_{6}\right)$ The following limit hold:

$$
\liminf _{s \rightarrow+\infty} \frac{s f(s)}{e^{\alpha_{0} s^{2}}}>\frac{4 e}{\alpha_{0}}
$$

The main result of this paper is stated as follows. 
Theorem 1.3. Suppose that $V$ satisfies $\left(V_{1}\right)-\left(V_{2}\right)$ and $f$ satisfies $\left(H_{1}\right)-$ $\left(H_{6}\right)$. Then, there exists $L^{*}=L^{*}\left(f, \mu, \alpha_{0}, \theta, a, b\right)>0$ such that equation (1.7) possesses a nontrivial weak solution $u \in E$ provided that $L_{a} \geq L^{*}$, namely $u \in E$ satisfies

$$
\int_{\mathbf{R}^{2}}(\nabla u \nabla \phi+V(x) u \phi) d x=\int_{\mathbf{R}^{2}} f(u) \phi d x, \quad \text { for all } \quad \phi \in E .
$$

In [9], under the hypotheses considered in that work, the potential $V$ can not vanish at infinity. Indeed, combining the fact that $V$ is a periodic function with $V(x) \rightarrow 0$, as $|x| \rightarrow \infty$, we obtain that $V \equiv 0$. Thus, 0 is not in the spectral gap of the operator $-\Delta \equiv-\Delta+V$.

We notice that in the equations (1.4), (1.5) and (1.6), it was assumed that the potential is bounded below for a positive constant. Thus, in [5, $10,13,11,8,12]$, the authors did not treat the case where $V$ can tend to zero at infinity or be zero somewhere.

In our work, the main difference with the above-mentioned results is that, by assumption $\left(V_{2}\right)$, the potential can vanish at infinity, and $V$ can be zero in $|x|<R_{0}$.

In order to find solutions of the equation (1.7), we combine a truncation argument with a finite-dimensional approximation and Mountain Pass Theorem.

The paper is organized as follows: Section 2 contains some preliminaries results. In section 3 , we set up an auxiliary functional and show that its energy functional associated has the mountain pass geometry. In section 4, we estimate the Palais-Smale sequences and minimax levels of the auxiliary functional. In section 5, we find a nontrivial critical point of the auxiliary functional. Finally, in sections 6 , we present the proof of our main result.

\section{Preliminaries}

In the first result of this section, we state the following lemma which proof can be found in [17, Lemma 2.1].

Lemma 2.1. Suppose that $\left(V_{1}\right)$ and $\left(V_{2}\right)$ hold. Then,

$$
|w(x)| \leq \frac{\|w\|}{L_{a}^{1 / 4} \pi^{1 / 2}|x|^{\frac{2-a}{4}}}, \quad \text { for all } \quad|x| \geq R_{0},
$$

for every $w \in E$. 
Let $\Omega$ is a bounded domain in $\mathbf{R}^{2}$. A famous result obtained independently by Pohožaev [16] and Trudinger [20] states that $e^{\alpha u^{2}} \in L^{1}(\Omega)$ for all $u \in H_{0}^{1}(\Omega)$ and $\alpha>0$. Furthermore, Moser [15] showed that there exists $C=C(\alpha)>0$ such that

$$
\sup _{u \in H_{0}^{1}(\Omega),\|\nabla u\|_{2} \leq 1} \int_{\Omega}\left(e^{\alpha u^{2}}-1\right) d x \leq C|\Omega| .
$$

Moreover, inequality (2.1) is sharp, in the sense that for any $\alpha>4 \pi$ the corresponding supremum becomes infinity.

In what follows, we will use the following version of the Trudinger-Moser inequality which is defined on the space $E$.

Proposition 2.2. (See [17]) Assume $V$ satisfies $\left(V_{1}\right)$ and $\left(V_{2}\right)$. Then,

$(2.2) \int_{\mathbf{R}^{2}}\left(e^{\alpha|u|^{2}}-\sum_{j=0}^{j_{a}} \frac{\alpha^{j}|u|^{2 j}}{j !}\right) d x<+\infty, \quad$ for all $\quad u \in E$ and $\alpha>0$

where $j_{a}=[|4 /(2-a)|]$. Furthermore, if $0<\alpha<4 \pi$, there exists a positive constant $C=C\left(\alpha, a, R_{0}\right)$ such that

$$
\sup _{u \in E,\|u\| \leq 1} \int_{\mathbf{R}^{2}}\left(e^{\alpha u^{2}}-\sum_{j=0}^{j_{a}} \frac{\alpha^{j}|u|^{2 j}}{j !}\right) d x \leq C .
$$

Lemma 2.3. Let $\alpha>0$ and $m>1$. Then, for each $n>m$ there exists a positive constant $C=C(n)$ such that

$$
\left(e^{\alpha|t|^{2}}-\sum_{j=0}^{j_{a}} \frac{\alpha^{j}|t|^{2 j}}{j !}\right)^{m} \leq C\left(e^{n \alpha|t|^{2}}-\sum_{j=0}^{j_{a}} \frac{n^{j} \alpha^{j}|t|^{2 j}}{j !}\right), \quad \text { for all } \quad t \in \mathbf{R}
$$

Proof. Since

$$
\lim _{|t| \rightarrow 0} \frac{\left(e^{\alpha|t|^{2}}-\sum_{j=0}^{j_{a}} \frac{\alpha^{j}|t|^{2 j}}{j !}\right)^{m}}{\left(e^{n \alpha|t|^{2}}-\sum_{j=0}^{j_{a}} \frac{n^{j} \alpha^{j}|t|^{2 j}}{j !}\right)}=0=\lim _{|t| \rightarrow \infty} \frac{\left(e^{\alpha|t|^{2}}-\sum_{j=0}^{j_{a}} \frac{\alpha^{j}|t|^{2 j}}{j !}\right)^{m}}{\left(e^{n \alpha|t|^{2}}-\sum_{j=0}^{j_{a}} \frac{n^{j} \alpha^{j}|t|^{2 j}}{j !}\right)}
$$

the conclusion follows. 


\section{The auxiliary functional}

Given $R>R_{0}$, we define a function $\tilde{f}: \mathbf{R}^{2} \times[0,+\infty) \rightarrow[0,+\infty)$ by

$$
\widetilde{f}(x, t)= \begin{cases}f(t), & |x| \leq R \\ \min \left\{f(t), V(x) t^{\mu-1}\right\}, & |x|>R\end{cases}
$$

where $\mu>b^{*}$ is given by $\left(H_{2}\right)$. Moreover, we set $\widetilde{f}(x, t)=0$ for $t \leq 0$.

Lemma 3.1. (See [17]) Suppose that $f$ satisfies $\left(H_{1}\right)$ and $\left(H_{2}\right)$. Then,

$$
0<\mu \widetilde{F}(x, t) \leq t \widetilde{f}(x, t), \quad \text { for all } t>0
$$

where $\mu>b^{*}$ is given by $\left(H_{2}\right)$ and $\widetilde{F}(x, t)=\int_{0}^{t} \widetilde{f}(x, s) d s$.

Using the function $\tilde{f}$, we consider the following auxiliary functional $\widetilde{J}: E \rightarrow \mathbf{R}$ defined by

$$
\widetilde{J}(u)=\int_{\mathbf{R}^{2}}\left(|\nabla u|^{2}+V(x) u^{2}\right) d x-\int_{\mathbf{R}^{2}} \widetilde{F}(x, u) d x, \quad \text { for all } \quad u \in E .
$$

Fix $1 \leq p<+\infty$. We consider the subspace $\Xi^{p}=H_{V, \text { rad }}^{1}\left(\mathbf{R}^{2}\right) \cap L_{V}^{p}\left(\mathbf{R}^{2}\right)$ endowed with the norm

$$
\|u\|_{\Xi^{p}}:=\|u\|_{H_{V}^{1}\left(\mathbf{R}^{2}\right)}+\|u\|_{L_{V}^{p}\left(\mathbf{R}^{2}\right)} .
$$

Lemma 3.2. (See [17]) If $u_{n} \rightarrow u$ in $\Xi^{p}$, then there exist a subsequence $\left(w_{n}\right)$ of $\left(u_{n}\right)$ and $g$ in $L_{V}^{p}\left(\mathbf{R}^{2}\right)$ such that, almost everywhere in $\mathbf{R}^{2}, w_{n}(x) \rightarrow$ $u(x)$ and

$$
|u(x)|,\left|w_{n}(x)\right| \leq g(x)
$$

Lemma 3.3. The functional $\widetilde{J}$ is well defined. Moreover, $\widetilde{J}$ belongs to $\mathcal{C}^{1}(E, \mathbf{R})$ and

$$
\widetilde{J}^{\prime}(u) \phi=\int_{\mathbf{R}^{2}}(\nabla u \nabla \phi+V(x) u \phi) d x-\int_{\mathbf{R}^{2}} \widetilde{f}(x, u) \phi d x,
$$

for all $u, \phi \in E$. 
Proof. Consider $\widetilde{J}_{1}: E \rightarrow \mathbf{R}$ given by

$$
\widetilde{J}_{1}(u)=\int_{\mathbf{R}^{2}}\left(|\nabla u|^{2}+V(x) u^{2}\right) d x .
$$

By definition of the space $E$, we have that the functional $\widetilde{J_{1}}$ is well defined. Moreover, let $B: E \times E \rightarrow \mathbf{R}$ given by

$$
B(u, v)=\int_{\mathbf{R}^{2}}(\nabla u \nabla v+V(x) u v) d x, \quad \text { for all } \quad(u, v) \in E \times E .
$$

Then, $B$ is a bilinear function. From Hölder's inequality, we have

$$
\begin{aligned}
|B(u, v)| \leq & \left(\int_{\mathbf{R}^{2}}|\nabla u|^{2} d x\right)^{1 / 2}\left(\int_{\mathbf{R}^{2}}|\nabla v|^{2} d x\right)^{1 / 2} \\
& +\left(\int_{\mathbf{R}^{2}} V(x) u^{2} d x\right)^{1 / 2}\left(\int_{\mathbf{R}^{2}} V(x) v^{2} d x\right)^{1 / 2} \\
\leq & 2\|u\|\|v\| .
\end{aligned}
$$

Thus, $B$ is a continuous bilinear function and $B \in \mathcal{C}^{\infty}(E \times E, \mathbf{R})$. Moreover, since $\widetilde{J}_{1}(u)=B(u, u)$, we conclude that $\widetilde{J}_{1} \in \mathcal{C}^{\infty}(E, \mathbf{R})$ and

$$
J_{1}^{\prime}(u) \phi=B(u, \phi)=\int_{\mathbf{R}^{2}}(\nabla u \nabla \phi+V(x) u \phi) d x, \quad \text { for all } u, \phi \in E .
$$

On the other hand, setting $\widetilde{J}_{F_{1}}: H^{1}\left(B_{R}\right) \rightarrow \mathbf{R}$ and $\widetilde{J}_{F_{2}}: \Xi^{\mu} \rightarrow \mathbf{R}$ defined by

$\widetilde{J}_{F_{1}}(u)=\int_{\mathbf{R}^{2}} \widetilde{F}(x, u) \chi_{B_{R}}(x) d x \quad$ and $\quad \widetilde{J}_{F_{2}}(u)=\int_{\mathbf{R}^{2}} \widetilde{F}(x, u)\left(1-\chi_{B_{R}}(x)\right) d x$.

We recall the existence of an extension operator $P: H^{1}\left(B_{R}\right) \rightarrow H^{1}\left(\mathbf{R}^{2}\right)$ such that $P u_{\mid B_{R}}=u$. Using the Trudinger-Moser's inequality in the whole space (see [4, Lemma 2.1]), for all $u \in H^{1}\left(B_{R}\right)$ and $\alpha>0$, we have

$$
\int_{B_{R}}\left(e^{\alpha|u|^{2}}-1\right) d x=\int_{B_{R}}\left(e^{\alpha|P u|^{2}}-1\right) d x \leq \int_{\mathbf{R}^{2}}\left(e^{\alpha|P u|^{2}}-1\right) d x<+\infty,
$$

which implies

$$
\int_{B_{R}} e^{\alpha|u|^{2}} d x<+\infty, \quad \text { for all } u \in H^{1}\left(B_{R}\right), \alpha>0 .
$$

We observe that

$$
\widetilde{J}_{F_{1}}(u)=\int_{B_{R}} \widetilde{F}(x, u) d x, \quad \text { for all } \quad u \in H^{1}\left(B_{R}\right) .
$$


From $\left(H_{1}\right)$ and $\left(H_{5}\right)$, for $\alpha>\alpha_{0}$ there exists $c>0$ such that

$$
f(s) \leq c e^{\alpha|s|^{2}}, \quad \text { for all } s \in \mathbf{R} .
$$

Thus, for $|x| \leq R$, we have

$$
|\widetilde{F}(x, t)|=\left|\int_{0}^{t} \widetilde{f}(x, s) d s\right| \leq \int_{0}^{t} f(s) d s \leq c \int_{0}^{|t|} e^{\alpha|s|^{2}} d s \leq \frac{c}{2}\left(e^{2 \alpha|t|^{2}}+|t|^{2}\right) .
$$

Using (3.3), (3.5) and the embedding of $H^{1}\left(B_{R}\right)$ in $L^{2}\left(B_{R}\right)$, we obtain

$$
\int_{B_{R}} \widetilde{F}(x, u) d x<+\infty, \quad \text { for all } u \in H^{1}\left(B_{R}\right) .
$$

Thus, $\widetilde{J}_{F_{1}}$ is well defined. Now, set $u, v \in H^{1}\left(B_{R}\right)$ and $0<|t|<1$. By the mean value theorem, there exists $\theta(x, t) \in(0,1)$ such that

$$
\frac{\widetilde{F}(x, u+t v)-\widetilde{F}(x, u)}{t}=\widetilde{f}(x, u+\theta(x, t) t v) v .
$$

Since the function $\widetilde{f}(x, t)$ is continuous in the second variable, it follows that

$$
\lim _{t \rightarrow 0} \frac{\widetilde{F}(x, u+t v)-\widetilde{F}(x, u)}{t}=\widetilde{f}(x, u) v .
$$

Moreover, using (3.4) in (3.6) and the fact that $\tilde{f}(x, t) \leq f(t)$, we get

$$
\left|\frac{\widetilde{F}(x, u+t v)-\widetilde{F}(x, u)}{t}\right| \leq c e^{\alpha(|u|+|v|)^{2}}|v| \leq \frac{c}{2}\left(e^{2 \alpha(|u|+|v|)^{2}}+|v|^{2}\right) \in L^{1}\left(B_{R}\right) .
$$

From Dominated convergence theorem, we find

$$
\begin{aligned}
\widetilde{J}_{F_{1}}^{\prime}(u) v & =\lim _{t \rightarrow 0} \frac{\widetilde{J}_{F_{1}}(u+t v)-\widetilde{J}_{F_{1}}(u)}{t} \\
& =\lim _{t \rightarrow 0} \int_{\Omega} \frac{\widetilde{F}(x, u+t v)-\widetilde{F}(x, u)}{t} d x \\
& =\int_{B_{R}} \lim _{t \rightarrow 0} \frac{\widetilde{F}(x, u+t v)-\widetilde{F}(u)}{t} d x \\
& =\int_{B_{R}} \widetilde{f}(x, u) v d x .
\end{aligned}
$$


In order to prove the continuity of $\widetilde{J}_{F_{1}}$, let $\left(u_{n}\right)$ be a sequence in $H^{1}\left(B_{R}\right)$ such that $u_{n} \rightarrow u$ in $H^{1}\left(B_{R}\right)$. Arguing similarly as Proposition 2.7 in [10], we can assume that $u_{n} \rightarrow u$ almost everywhere in $B_{R}$ and there exists $v \in H^{1}\left(B_{R}\right)$ such that $\left|u_{n}(x)\right| \leq v(x)$ almost everywhere in $B_{R}$. Consequently,

$$
\left|\widetilde{f}\left(x, u_{n}\right)-\widetilde{f}(x, u)\right|^{2} \leq 2 c\left(e^{2 \alpha|v|^{2}}+e^{2 \alpha|u|^{2}}\right) \in L^{1}\left(B_{R}\right)
$$

by the continuity of $\tilde{f}$ almost everywhere in $B_{R}$, we get

$$
\left|\widetilde{f}\left(x, u_{n}\right)-\widetilde{f}(x, u)\right|^{2} \rightarrow 0, \quad \text { almost everywhere in } \quad B_{R} .
$$

By Lebesgue's dominated convergence theorem, we obtain

$$
\begin{aligned}
\left\|\widetilde{J}_{F_{1}}^{\prime}\left(u_{n}\right)-\widetilde{J}_{F_{1}}^{\prime}(u)\right\| & =\sup _{\|v\|_{H^{1}\left(B_{R}\right)} \leq 1}\left|\left\langle\widetilde{J}_{F_{1}}^{\prime}\left(u_{n}\right)-\widetilde{J}_{F_{1}}^{\prime}(u), v\right\rangle\right| \\
& =\sup _{\|v\|_{H^{1}\left(B_{R}\right)} \leq 1}\left|\int_{B_{R}}\left(\widetilde{f}\left(x, u_{n}\right)-\widetilde{f}(x, u)\right) v d x\right| \\
& \leq \sup _{\|v\|_{H^{1}\left(B_{R}\right)} \leq 1}\left\|\widetilde{f}\left(x, u_{n}\right)-\widetilde{f}(x, u)\right\|_{L^{2}\left(B_{R}\right)}\|v\|_{L^{2}\left(B_{R}\right)} \\
& =o_{n}(1) .
\end{aligned}
$$

Thus, $\widetilde{J}_{F_{1}} \in \mathcal{C}^{1}\left(H^{1}\left(B_{R}\right), \mathbf{R}\right)$. Since $E \hookrightarrow H^{1}\left(B_{R}\right)$ continuously, it follows that $\widetilde{J}_{F_{1}} \in \mathcal{C}^{1}(E, \mathbf{R})$ and

$$
\widetilde{J}_{F_{1}}^{\prime}(u) \phi=\int_{B_{R}} \tilde{f}(x, u) \phi d x, \quad \text { for all } u, \phi \in E .
$$

Notice that $\widetilde{F}(x, s)\left(1-\chi_{B_{R}}(x)\right)$ is a Carathéodory function in $(x, s) \in$ $\mathbf{R}^{2} \times \mathbf{R}$ and

$$
\left|\widetilde{f}(x, s)\left(1-\chi_{B_{R}}(x)\right)\right| \leq V(x)|s|^{\mu-1}, \quad \text { for all } \quad(x, s) \in \mathbf{R}^{2} \times \mathbf{R} .
$$

Using Lemma 3.2 and arguing similarly as Lemma 17.1 in [14], we have $\widetilde{J}_{F_{2}} \in \mathcal{C}^{1}\left(\Xi^{\mu}, \mathbf{R}\right)$, by Lemma 1.1 , the embedding $E \hookrightarrow \Xi^{\mu}$ is continuous, we have $\widetilde{J}_{F_{2}} \in \mathcal{C}^{1}(E, \mathbf{R})$ and

$$
\widetilde{J}_{F_{2}}^{\prime}(u) \phi=\int_{\mathbf{R}^{2} \backslash B_{R}} \tilde{f}(x, u) \phi d x, \quad \text { for all } \quad u, \phi \in E .
$$

Finally, note that $\widetilde{J}=\widetilde{J}_{1}-\widetilde{J}_{F_{1}}-\widetilde{J}_{F_{2}}$. Thus, $\widetilde{J}$ is well defined and using (3.2), (3.7) and (3.8), we obtain (3.1). 


\subsection{The geometry of Mountain Pass}

This section is devoted to set the geometry of the Mountain Pass Theorem of the auxiliary functional.

Lemma 3.4. Suppose $\left(V_{1}\right),\left(V_{2}\right),\left(H_{1}\right),\left(H_{4}\right)$ and $\left(H_{5}\right)$ hold. Then, there exist $\sigma>0$ and $\rho>0$ such that

$$
\widetilde{J}(u) \geq \sigma, \quad \text { for all } \quad u \in E, \quad\|u\|=\rho .
$$

Proof. From $\left(H_{4}\right)$, we have $f(s)=o\left(s^{a^{*}-1}\right)$. Thus, there exists $\delta_{0}>0$ such that

$$
|f(s)| \leq|s|^{a^{*}-1}, \quad \text { for all }|s|<\delta_{0} .
$$

By $\left(H_{5}\right)$, we can find constants $c>0, \delta_{1}>\delta_{0}$ and $q \geq a^{*}$ such that

$$
|f(s)| \leq c|s|^{q-1}\left(e^{2 \alpha_{0}|s|^{2}}-\sum_{j=0}^{j_{a}} \frac{2^{j} \alpha_{0}^{j}|s|^{2 j}}{j !}\right), \text { for all }|s| \geq \delta_{1} .
$$

Note also that for all $\delta_{0} \leq|s| \leq \delta_{1}$, we have

$$
|f(s)| \leq \frac{|s|^{q-1}\left(e^{2 \alpha_{0}|s|^{2}}-\sum_{j=0}^{j_{a}} \frac{2^{j} \alpha_{0}^{j}|s|^{2 j}}{j !}\right)}{\left|\delta_{0}\right|^{q-1}\left(e^{2 \alpha_{0}\left|\delta_{0}\right|^{2}}-\sum_{j=0}^{j_{a}} \frac{2^{j} \alpha_{0}^{j}\left|\delta_{0}\right|^{2 j}}{j !}\right)} \max _{\delta_{0} \leq|s| \leq \delta_{1}}|f(s)| .
$$

From these estimates, we get a constant $c>0$ such that

$$
|f(s)| \leq|s|^{a^{*}-1}+c|s|^{q-1}\left(e^{2 \alpha_{0}|s|^{2}}-\sum_{j=0}^{j_{a}} \frac{2^{j} \alpha_{0}^{j}|s|^{2 j}}{j !}\right), \quad \text { for all } \quad s \in \mathbf{R} .
$$

Then,

$$
|\widetilde{F}(x, s)| \leq|F(s)| \leq|s|^{a^{*}}+c|s|^{q}\left(e^{2 \alpha_{0}|s|^{2}}-\sum_{j=0}^{j_{a}} \frac{2^{j} \alpha_{0}^{j}|s|^{2 j}}{j !}\right), \quad \text { for all } \quad s \in \mathbf{R}
$$

By Lemma 2.3 and Proposition 2.2, we obtain

$$
\begin{aligned}
\int_{\mathbf{R}^{2}}|u|^{q}\left(e^{2 \alpha_{0}|u|^{2}}\right. & \left.-\sum_{j=0}^{j_{a}} \frac{2^{j} \alpha_{0}^{j}|u|^{2 j}}{j !}\right) d x \\
& \leq\|u\|_{2 q}^{q}\left(\int_{\mathbf{R}^{2}}\left(e^{2 \alpha_{0}|u|^{2}}-\sum_{j=0}^{j_{a}} \frac{2^{j} \alpha_{0}^{j}|u|^{2 j}}{j !}\right)^{2} d x\right)^{1 / 2} \\
& \leq c\|u\|_{2 q}^{q}\left(\int_{\mathbf{R}^{2}}\left(e^{6 \alpha_{0}|u|^{2}}-\sum_{j=0}^{j_{a}} \frac{6^{j} \alpha_{0}^{j}|u|^{2 j}}{j !}\right) d x\right)^{1 / 2} \\
& \leq c\|u\|_{2 q}^{q},
\end{aligned}
$$


provided that $\|u\| \leq \rho_{1}$ for some $\rho_{1}>0$ such that $6 \alpha_{0} \rho_{1}^{2}<4 \pi$.

Thus,

$$
\int_{\mathbf{R}^{2}} \widetilde{F}(x, u) d x \leq c\|u\|_{a^{*}}^{a^{*}}+c\|u\|_{2 q}^{q} .
$$

By Lemma 1.1, we obtain

$$
\widetilde{J}(u) \geq\|u\|^{2}-\int_{\mathbf{R}^{2}} \widetilde{F}(x, u) d x \geq\|u\|^{2}-c\|u\|^{a^{*}}-c\|u\|^{q} .
$$

Therefore, we can find $\rho>0$ and $\sigma>0$ with $\rho$ sufficiently small such that $\widetilde{J}(u) \geq \sigma$, for all $u \in E$ with $\|u\|=\rho$.

Lemma 3.5. Suppose that $\left(V_{1}\right)-\left(V_{2}\right)$ and $\left(H_{1}\right)-\left(H_{2}\right)$ hold. Then, there exists $e \in E$ such that

$$
\widetilde{J}(e)<\rho \text { and }\|e\|>\rho .
$$

where $\rho>0$ is given by Lemma 3.4.

Proof. It follows from Lemma 3.1 the existence of $c>0$ and $\vartheta>2$ such that

$$
\widetilde{F}(x, s) \geq c|s|^{\vartheta}-s^{2}, \text { for all }(x, s) \in \bar{B}_{1}(0) \times[0,+\infty) .
$$

Let $0 \neq e_{k_{0}} \in E$ fixed. Then,

$$
\begin{aligned}
\widetilde{J}\left(t e_{k_{0}}\right) & =t^{2}\left\|e_{k_{0}}\right\|^{2}-\int_{\mathbf{R}^{2}} \widetilde{F}\left(x, t e_{k_{0}}\right) d x \\
& \leq t^{2}\left\|e_{k_{0}}\right\|^{2}+\int_{B_{1}}\left(\left|t e_{k_{0}}\right|^{2}-c\left|t e_{k_{0}}\right|^{\vartheta}\right) d x \\
& \leq t^{2}\left\|e_{k_{0}}\right\|^{2}+t^{2}\left\|e_{k_{0}}\right\|_{2}^{2}-c t^{\vartheta}\left\|e_{k_{0}}\right\|_{\vartheta}^{\vartheta}
\end{aligned}
$$

Since, $\vartheta>2$. Then, $\widetilde{J}\left(t e_{k_{0}}\right) \rightarrow-\infty$. Thus, we can take $e=t_{0} e_{k_{0}}$ with $t_{0}>0$ sufficiently large such that $\widetilde{J}(e)<0$ and $\|e\|>\rho$.

By Lemmas 3.4 and 3.5 in Mountain Pass Theorem (see [21, Theorem 1.15]) and Ekeland's variational principle (see [21, Theorem 2.4]), there exists a Palais-Smale sequence at level $d \geq \sigma$, where $\sigma$ is given by Lemma 3.4 , that is, there exists a sequence $\left(u_{n}\right) \subset E$ such that

$$
\widetilde{J}\left(u_{n}\right) \rightarrow d \quad \text { and } \quad\left\|\widetilde{J}^{\prime}\left(u_{n}\right)\right\|_{E^{-}} \rightarrow 0,
$$

and $d>0$ can be characterized as

$$
d=\inf _{\gamma \in \Gamma} \max _{t \in[0,1]} \widetilde{J}(\gamma(t)),
$$


where

$$
\Gamma=\{\gamma \in \mathcal{C}([0,1], E): \gamma(0)=0, \gamma(1)=e\} .
$$

Lemma 3.6. Let $\left(u_{n}\right) \subset E$ be a Palais-Smale sequence satisfying (3.9).

Then, $\left\|u_{n}\right\| \leq c$, for every $n \in \mathbf{N}$ and for some positive constant $c$.

Proof. From Lemma 3.1, we obtain

$$
\begin{gathered}
J\left(u_{n}\right)-\frac{1}{\mu} \widetilde{J}^{\prime}\left(u_{n}\right) u_{n}=\left(\frac{1}{2}-\frac{1}{\mu}\right)\left\|u_{n}\right\|^{2}-\frac{1}{\mu} \int_{\mathbf{R}^{2}}\left(\mu \widetilde{F}\left(x, u_{n}\right)-\widetilde{f}\left(x, u_{n}\right) u_{n}\right) d x \\
\geq\left(\frac{1}{2}-\frac{1}{\mu}\right)\left\|u_{n}\right\|^{2} .
\end{gathered}
$$

Using (3.9), for $n$ sufficiently large, we have

$$
\widetilde{J}\left(u_{n}\right) \leq d+1 \text { and }\left\|\widetilde{J}^{\prime}\left(u_{n}\right)\right\|_{E^{-}} \leq \mu,
$$

Thus, for $n$ sufficiently large, we get

$$
\left|\widetilde{J}\left(u_{n}\right)-\frac{1}{\mu} \widetilde{J}^{\prime}\left(u_{n}\right) u_{n}\right| \leq d+1+\left\|u_{n}\right\| .
$$

Then, for $n$ sufficiently large, we obtain

$$
\left(\frac{1}{2}-\frac{1}{\mu}\right)\left\|u_{n}\right\|^{2} \leq d+1+\left\|u_{n}\right\|
$$

which implies that the sequence $\left(u_{n}\right)$ is bounded.

Lemma 3.7. (See [5, Lemma 2.1]) Let $\Omega$ be a bounded subset in $\mathbf{R}^{N}$, $f: \bar{\Omega} \times \mathbf{R} \rightarrow \mathbf{R}$ a continuous function and $\left(u_{n}\right)$ be a sequence of functions in $L^{1}(\Omega)$ converging to $u$ in $L^{1}(\Omega)$. Assume that $f(x, u(x))$ and $f\left(x, u_{n}(x)\right)$ are also $L^{1}(\Omega)$ functions. If

$$
\int_{\Omega}\left|f\left(x, u_{n}\right) u_{n}\right| d x \leq C,
$$

then, $f\left(x, u_{n}\right)$ converges in $L^{1}(\Omega)$ to $f(x, u)$.

Lemma 3.8. Let $\left(u_{n}\right)$ be a Palais-Smale sequence satisfying (3.9) and suppose that $u_{n} \rightarrow u$ in $E$. Then, there exists a subsequence still denoted by $\left(u_{n}\right)$ such that

$$
\tilde{f}\left(x, u_{n}\right) \rightarrow \tilde{f}(x, u) \quad \text { in } \quad L^{1}\left(B_{R_{1}}\right),
$$

where $R_{1}>R_{0}$ and

$$
\widetilde{F}\left(x, u_{n}\right) \rightarrow \widetilde{F}(x, u) \quad \text { in } \quad L^{1}\left(\mathbf{R}^{2}\right) .
$$


Proof. According to Remark 1.2, we can assume that $u_{n} \rightarrow u$ in $L^{1}\left(B_{R_{1}}\right)$. Moreover, by the exponential growth of $f$ and Proposition 2.2, we have that $\widetilde{f}\left(x, u_{n}\right) \in L^{1}\left(B_{R_{1}}\right)$. By Lemma 3.6, the sequence $\left(\left\|u_{n}\right\|\right)$ is bounded and since $\left\|\widetilde{J}^{\prime}\left(u_{n}\right)\right\|_{E^{-}} \rightarrow 0$, we obtain

$$
\left|\widetilde{J}^{\prime}\left(u_{n}\right) u_{n}\right| \leq\left\|\widetilde{J}^{\prime}\left(u_{n}\right)\right\|_{E^{-}}\left\|u_{n}\right\| \rightarrow 0 .
$$

Thus,

$$
\widetilde{J}^{\prime}\left(u_{n}\right) u_{n}=\left\|u_{n}\right\|^{2}-\int_{\mathbf{R}^{2}} \widetilde{f}\left(x, u_{n}\right) u_{n} d x \rightarrow 0 .
$$

Then, there exists $c>0$ such that

$$
\int_{\mathbf{R}^{2}} \widetilde{f}\left(x, u_{n}\right) u_{n} d x \leq c .
$$

Using Lemma 3.7, we conclude that $\tilde{f}\left(x, u_{n}\right) \rightarrow \widetilde{f}(x, u)$ in $L^{1}\left(B_{R_{1}}\right)$.

On the other hand, by the first part, given $R_{1} \geq R$, where $R$ is given by the definition of $\widetilde{f}$, we obtain

$$
\int_{B_{R_{1}}} \tilde{f}\left(x, u_{n}\right) d x \rightarrow \int_{B_{R_{1}}} \tilde{f}(x, u) d x
$$

Thus, there exists $p \in L^{1}\left(B_{R_{1}}\right)$ such that

$$
f\left(u_{n}\right) \leq p(x), \quad \text { almost everywhere in } \quad B_{R_{1}} .
$$

From $\left(H_{1}\right)$ and $\left(H_{3}\right)$, we obtain

$$
F(t) \leq \max _{t \in\left[0, s_{0}\right]} F(t)+M f(t), \quad \text { for all } \quad t \in \mathbf{R} .
$$

Using (3.11) and (3.12), we have

$$
\widetilde{F}\left(x, u_{n}\right) \leq F\left(u_{n}\right) \leq \max _{t \in\left[0, s_{0}\right]} F(t)+M p(x), \quad \text { almost everywhere in } \quad B_{R_{1}} .
$$

By Lebesgue's dominated convergence theorem, we obtain

$$
\int_{B_{R_{1}}} \widetilde{F}\left(x, u_{n}\right) d x \rightarrow \int_{B_{R_{1}}} \widetilde{F}(x, u) d x .
$$

Consequently, to prove that

$$
\int_{\mathbf{R}^{2}} \widetilde{F}\left(x, u_{n}\right) d x \rightarrow \int_{\mathbf{R}^{2}} \widetilde{F}(x, u) d x
$$


it is sufficient to show that given $\delta>0$, there exists $R_{1}>0$ such that

$$
\int_{\mathbf{R}^{2} \backslash B_{R_{1}}} \tilde{F}\left(x, u_{n}\right) d x<\delta \text { and } \int_{\mathbf{R}^{2} \backslash B_{R_{1}}} \tilde{F}(x, u) d x<\delta .
$$

Using definition of the function $\tilde{f}$, we have

$$
\widetilde{f}(x, t) \leq V(x) t^{\mu-1}, \quad \text { for all } \quad x \in \mathbf{R}^{2} \backslash B_{R_{1}} .
$$

Then, for all $x \in \mathbf{R}^{2} \backslash B_{R_{1}}$ and $t>0$, we have

$$
\widetilde{F}(x, t)=\int_{0}^{t} \widetilde{f}(x, s) d x \leq \int_{0}^{t} V(x) s^{\mu-1} d s=\frac{1}{\mu} V(x) t^{\mu} .
$$

Thus,

$$
\widetilde{F}\left(x, u_{n}\right) \leq \frac{1}{\mu} V(x)\left|u_{n}\right|^{\mu}, \quad \text { for all } \quad x \in \mathbf{R}^{2} \backslash B_{R_{1}} .
$$

Hence,

$$
\begin{aligned}
\int_{\mathbf{R}^{2} \backslash B_{R_{1}}} & \widetilde{F}\left(x, u_{n}\right) d x \leq \frac{1}{\mu} \int_{\mathbf{R}^{2} \backslash B_{R_{1}}} V(x)\left|u_{n}\right|^{\mu} d x \\
& \leq \frac{2^{\mu-1}}{\mu}\left(\int_{\mathbf{R}^{2} \backslash B_{R_{1}}} V(x)\left|u_{n}-u\right|^{\mu} d x+\int_{\mathbf{R}^{2} \backslash B_{R_{1}}} V(x)|u|^{\mu} d x\right) .
\end{aligned}
$$

Using the compactness of the embedding $E \hookrightarrow L_{V, r a d}^{\mu}\left(\mathbf{R}^{2}\right)$ and the weak convergence $u_{n} \rightarrow u$ in $E$, we can choose $R_{1}>0$ sufficiently large such that

$$
\int_{\mathbf{R}^{2} \backslash B_{R_{1}}} \widetilde{F}\left(x, u_{n}\right) d x<\delta .
$$

Since $\widetilde{F}(\cdot, u) \in L^{1}\left(\mathbf{R}^{2}\right)$, we may assume that

$$
\int_{\mathbf{R}^{2} \backslash B_{R_{1}}} \widetilde{F}(x, u) d x<\delta
$$

Combining all the above estimates, since $\delta>0$ is arbitrary, we have

$$
\int_{\mathbf{R}^{2}} \widetilde{F}\left(x, u_{n}\right) d x \rightarrow \int_{\mathbf{R}^{2}} \widetilde{F}(x, u) d x
$$




\section{Estimates}

In this section, we establish the estimates for the auxiliary functional that are used to prove Proposition 5.1. We start with the definition of Moser type functions. Consider $k \in \mathbf{N}$. Let $\delta_{k}>0$ be a sequence which will be fixed such that $\delta_{k} \rightarrow 0$, as $k \rightarrow+\infty$. The Moser type functions are defined by

$$
e_{k}=\frac{1}{\sqrt{2 \pi}} \begin{cases}\sqrt{\ln k}\left(1-\delta_{k}\right)^{1 / 2}, & |x| \leq \frac{1}{k}, \\ \ln \left(\frac{1}{|x|}\right) \frac{\left(1-\delta_{k}\right)^{1 / 2}}{\sqrt{\ln k},} & \frac{1}{k}<|x| \leq 1, \\ 0, & |x|>1 .\end{cases}
$$

Therefore,

$$
\left\|\nabla e_{k}\right\|_{2}^{2}=1-\delta_{k}
$$

and

$$
\int_{\mathbf{R}^{2}} V(x) e_{k}^{2} d x \leq\left(1-\delta_{k}\right)\left(\frac{\ln k}{k^{2}}+\frac{1}{4 \ln k}\right) .
$$

Then, we may choose $\delta_{k}$, depending on $k$ such that

$$
\left\|e_{k}\right\|=1, \quad \text { for all } \quad k \geq 1 \text {. }
$$

Furthermore, we can see that

$$
\delta_{k} \leq\left(1-\delta_{k}\right)\left(\frac{\ln k}{k^{2}}+\frac{1}{4 \ln k}\right) \leq\left(\frac{\ln k}{k^{2}}+\frac{1}{4 \ln k}\right) .
$$

Thus,

$$
\delta_{k} \ln k \leq \frac{1}{2}, \quad \text { for } k \text { sufficiently large } .
$$

Proposition 4.1. Suppose that $\left(H_{1}\right)-\left(H_{6}\right)$ hold. Then, there exists $k_{0} \in$ $\mathbf{N}$ such that

$$
\max \left\{\widetilde{J}\left(t e_{k_{0}}\right): t \geq 0\right\}<\frac{2 \pi}{\alpha_{0}} .
$$

Proof. Suppose, by contradiction, that for all $k \in \mathbf{N}$

$$
\max \left\{\widetilde{J}\left(t e_{k}\right): t \geq 0\right\} \geq \frac{2 \pi}{\alpha_{0}} .
$$

Thus, for all fixed $k \geq 1$, there exists $t_{k}>0$ such that

$$
\widetilde{J}\left(t_{k} e_{k}\right)=\max \left\{\widetilde{J}\left(t e_{k}\right): t \geq 0\right\} \geq \frac{2 \pi}{\alpha_{0}} .
$$


Then,

$$
\widetilde{J}\left(t_{k} e_{k}\right)=\frac{t_{k}^{2}\left\|e_{k}\right\|^{2}}{2}-\int_{\mathbf{R}^{2}} \widetilde{F}\left(x, t e_{k}\right) d x \geq \frac{2 \pi}{\alpha_{0}} .
$$

and

$$
\frac{d}{d t} \widetilde{J}\left(t e_{k}\right)=0, \quad \text { in } \quad t=t_{k}
$$

That is,

$$
t_{k}\left\|e_{k}\right\|^{2}-\int_{\mathbf{R}^{2}} \tilde{f}\left(x, t_{k} e_{k}\right) e_{k} d x=0 .
$$

Using last equations and the fact that $\left\|e_{k}\right\|=1$, we have

$$
t_{k}^{2} \geq \frac{4 \pi}{\alpha_{0}}
$$

and

$$
t_{k}^{2}=\int_{\mathbf{R}^{2}} \widetilde{f}\left(x, t_{k} e_{k}\right) t_{k} e_{k} d x
$$

Set $l>0$ such that

$$
\liminf _{t \rightarrow+\infty} \frac{t f(t)}{e^{\alpha_{0} t^{2}}}>l>\frac{4 e}{\alpha_{0}} .
$$

Thus, given $\epsilon>0$, there exists $R_{\epsilon}>0$ such that

$$
t f(t) \geq(l-\epsilon) e^{\alpha_{0} t^{2}}, \quad \text { for all } t \geq R_{\epsilon} .
$$

Using the fact that $\left(t_{k}\right)$ is bounded below, there exists $k_{0}>0$ such that

$$
\left(1-\delta_{k}\right)^{1 / 2} t_{k} \frac{\sqrt{\ln k}}{2 \pi} \geq R_{\epsilon}, \quad \text { for all } \quad k \geq k_{0} .
$$

Since,

$$
e_{k}(x)=\left(1-\delta_{k}\right)^{1 / 2} \sqrt{\frac{\ln k}{2 \pi}}, \quad \text { for all } \quad x \in B_{1 / k}
$$

we get

$$
\begin{aligned}
t_{k}^{2} & =\int_{\mathbf{R}^{2}} \tilde{f}\left(x, t_{k} e_{k}\right) t_{k} e_{k} d x \\
& \geq \int_{B_{1 / k}} \tilde{f}\left(x, t_{k} e_{k}\right) t_{k} e_{k} d x \\
& =\int_{B_{1 / k}} f\left(t_{k} e_{k}\right) t_{k} e_{k} d x \\
& \geq(l-\epsilon) \int_{B_{1 / k}} e^{\alpha_{0}\left(1-\delta_{k}\right) \frac{\ln k}{2 \pi} t_{k}^{2}} d x
\end{aligned}
$$


for every $k \geq k_{0}$. Define $s_{k}:=t_{k}^{2}-\frac{4 \pi}{\alpha_{0}}$. Then,

$$
\begin{aligned}
\frac{4 \pi}{\alpha_{0}}+s_{k} & \geq(l-\epsilon) \int_{B_{1 / k}} e^{\alpha_{0}\left(1-\delta_{k}\right) \frac{\ln k}{2 \pi}\left(\frac{4 \pi}{\alpha_{0}}+s_{k}\right)} d x \\
& =(l-\epsilon) e^{\alpha_{0} \frac{\ln k}{2 \pi}\left(\frac{4 \pi}{\alpha_{0}}+s_{k}\right)} e^{-\frac{\alpha_{0}}{2 \pi}\left(\frac{4 \pi}{\alpha_{0}}+s_{k}\right) \delta_{k} \ln k} \int_{1 / k} 1 d x \\
& =(l-\epsilon) e^{2 \ln k} e^{\alpha_{0} \frac{\ln k}{2 \pi} s_{k}} e^{-\frac{\alpha_{0}}{2 \pi}\left(\frac{4 \pi}{\alpha_{0}}+s_{k}\right) \delta_{k} \ln k} \frac{\pi}{k^{2}} \\
& =(l-\epsilon) \pi e^{\alpha_{0} \frac{\ln k}{2 \pi} s_{k}} e^{-\frac{\alpha_{0}}{2 \pi}\left(\frac{4 \pi}{\alpha_{0}}+s_{k}\right) \delta_{k} \ln k}
\end{aligned}
$$

for every $k \geq k_{0}$. Using (4.1), we obtain

$$
\frac{4 \pi}{\alpha_{0}}+s_{k} \geq(l-\epsilon) \pi e^{\alpha_{0} \frac{\ln k}{2 \pi} s_{k}} e^{-\frac{\alpha_{0}}{4 \pi}\left(\frac{4 \pi}{\alpha_{0}}+s_{k}\right)} .
$$

Thus,

$$
\frac{4 \pi}{\alpha_{0}}+s_{k} \geq(l-\epsilon) \pi e^{\frac{\alpha_{0} s_{k}}{2 \pi}(\ln k-1 / 2)} e^{-1} .
$$

Inequality (4.5) implies that $\left(s_{k}\right)$ is bounded for each $k \geq k_{0}$. Therefore, there exists $s \in \mathbf{R}$ such that $\lim \sup s_{k}=s$. By (4.2), $s \geq 0$. Using the last limit in (4.5) and taking $k \rightarrow+\infty \rightarrow \infty$, we see that necessarily $s=0$. Then, $\lim _{k \rightarrow \infty} s_{k}=0$. Using this in (4.5), yields

$$
\frac{4 \pi}{\alpha_{0}} \geq(l-\epsilon) \pi e^{-1}
$$

This contradicts (4.3) because $\epsilon>0$ is arbitrary.

Remark 4.2. Taking $e_{k_{0}}$ given by Proposition 4.1 in Lemma 3.5. Thus, $e=t_{0} e_{k_{0}}$. Define $\gamma_{0}(t)=t t_{0} e_{k_{0}}$. Then, $\gamma_{0} \in \Gamma=\{\gamma \in \mathcal{C}([0,1], E): \gamma(0)=$ $0, \gamma(1)=e\}$. By Proposition 4.1 and (3.10), we obtain

$d=\inf _{\gamma \in \Gamma} \max _{t \in[0,1]} \widetilde{J}(\gamma(t)) \leq \max _{t \in[0,1]} \widetilde{J}\left(\gamma_{0}(t)\right) \leq \max _{t \in[0,1]} \widetilde{J}\left(t t_{0} e_{k_{0}}\right) \leq \max _{t \geq 0} \widetilde{J}\left(t e_{k_{0}}\right)<\frac{2 \pi}{\alpha_{0}}$

\section{Existence of critical point of the auxiliary functional}

Proposition 5.1. Suppose that $V$ satisfies $\left(V_{1}\right)-\left(V_{2}\right)$ and $f$ satisfies $\left(H_{1}\right)-\left(H_{6}\right)$. Then, $\widetilde{J}$ possesses a nontrivial critical point. 
Proof. Let $\left(u_{n}\right) \subset E$ be a sequence satisfying (3.9). Then,

$$
\widetilde{J}^{\prime}\left(u_{n}\right) \phi=\int_{\mathbf{R}^{2}}\left(\nabla u_{n} \nabla \phi+V(x) u_{n} \phi\right) d x-\int_{\mathbf{R}^{2}} \widetilde{f}\left(x, u_{n}\right) \phi d x=o_{n}(1),
$$

for all $\phi \in \mathcal{C}_{0, r}^{\infty}\left(\mathbf{R}^{2}\right)$.

By Lemma 3.6, the sequence $\left(u_{n}\right)$ is bounded in $E$. Thus, we can assume that there exists $u \in E$ such that $u_{n} \rightarrow u$ in $E$, using this together with Lemma 5.2 in (5.1), we obtain passing to limit

$$
\int_{\mathbf{R}^{2}}(\nabla u \nabla \phi+V(x) u \phi) d x-\int_{\mathbf{R}^{2}} \widetilde{f}(x, u) \phi d x=0, \quad \text { for all } \quad \phi \in \mathcal{C}_{0, r}^{\infty}\left(\mathbf{R}^{2}\right)
$$

Using the fact that $\mathcal{C}_{0, \text { rad }}^{\infty}\left(\mathbf{R}^{2}\right)$ is dense in $E$, yields

$$
\int_{\mathbf{R}^{2}}(\nabla u \nabla \phi+V(x) u \phi) d x=\int_{\mathbf{R}^{2}} \widetilde{f}(x, u) \phi d x, \quad \text { for all } \quad \phi \in E .
$$

Thus, $u \in E$ is a critical point of $\widetilde{J}$. To conclude the proof, it only remains to prove that $u$ is nontrivial. Suppose, by contradiction, that $u \equiv 0$. From Lemma 1.1, we can assume that

$$
u_{n} \rightarrow 0 \quad \text { in } L_{V, r a d}^{r}\left(\mathbf{R}^{2}\right), \quad \text { for all } r>b^{*} .
$$

Using the fact that $\widetilde{J}\left(u_{n}\right) \rightarrow d$, we have

$$
\widetilde{J}\left(u_{n}\right)=\frac{\left\|u_{n}\right\|^{2}}{2}-\int_{\mathbf{R}^{2}} \widetilde{F}\left(x, u_{n}\right) d x=d+o_{n}(1) .
$$

Since, we suppose that $u_{n} \rightarrow 0$, by Lemma 3.8, we obtain

$$
\int_{\mathbf{R}^{2}} \widetilde{F}\left(x, u_{n}\right) d x \rightarrow \int_{\mathbf{R}^{2}} \widetilde{F}(x, 0) d x=0 .
$$

Replacing in (5.3), we have

$$
\frac{\left\|u_{n}\right\|^{2}}{2}=d+o_{n}(1)
$$

By Remark 4.2, we get

$$
\left\|u_{n}\right\|^{2}=2 d+o_{n}(1)<\frac{4 \pi}{\alpha_{0}}+o_{n}(1) .
$$


Thus, we can assume that there exists $\delta>0$ sufficiently small such that

$$
\left\|u_{n}\right\|^{2} \leq \frac{4 \pi}{\alpha_{0}}-\delta, \quad \text { for all } n \text { sufficiently large. }
$$

Taking $p>1$ sufficiently close to 1 and $\epsilon>0$ sufficiently small such that

$$
p\left(\alpha_{0}+\epsilon\right)\left(\frac{4 \pi}{\alpha_{0}}-\delta\right)<4 \pi
$$

From the continuity and the critical growth of $f$ there exists a positive constant $C$ such that

$$
|f(s)| \leq|s|^{a^{*}-1}+C\left(e^{\left(\alpha_{0}+\epsilon\right)|s|^{2}}-\sum_{j=0}^{j_{a}} \frac{\left(\alpha_{0}+\epsilon\right)^{j}|s|^{2 j}}{j !}\right), \quad \text { for all } \quad s \in \mathbf{R} .
$$

By Hölder's inequality and (5.6), we have

$$
\begin{gathered}
\int_{B_{R}} \tilde{f}\left(x, u_{n}\right) u_{n} d x=\int_{B_{R}} f\left(u_{n}\right) u_{n} d x \\
\leq\left\|u_{n}\right\|_{a^{*}}^{a^{*}}+C \int_{B_{R}}\left(e^{\left(\alpha_{0}+\epsilon\right)\left|u_{n}\right|^{2}}-\sum_{j=0}^{j_{a}} \frac{\left(\alpha_{0}+\epsilon\right)^{j}\left|u_{n}\right|^{2 j}}{j !}\right)\left|u_{n}\right| d x \\
\leq\left\|u_{n}\right\|_{a^{*}}^{a^{*}}+C\left\|u_{n}\right\|_{p^{\prime}}\left(\int_{B_{R}}\left(e^{\left(\alpha_{0}+\epsilon\right)\left|u_{n}\right|^{2}}-\sum_{j=0}^{j_{a}} \frac{\left(\alpha_{0}+\epsilon\right)^{j}\left|u_{n}\right|^{2 j}}{j !}\right)^{p} d x\right)^{1 / p} .
\end{gathered}
$$

From (5.5), we can find for some $p_{0}>p$ such that

$$
p_{0}\left(\alpha_{0}+\epsilon\right)\left(\frac{4 \pi}{\alpha_{0}}-\delta\right)<4 \pi
$$

Using Lemma 2.3 in the last integral of (5.7) there exists $C_{0}>0$ such that

$$
\begin{aligned}
\int_{B_{R}} & \left(e^{\left(\alpha_{0}+\epsilon\right)\left|u_{n}\right|^{2}}-\sum_{j=0}^{j_{a}} \frac{\left(\alpha_{0}+\epsilon\right)^{j}\left|u_{n}\right|^{2 j}}{j !}\right)^{p} d x \\
& \leq C_{0} \int_{B_{R}}\left(e^{p_{0}\left(\alpha_{0}+\epsilon\right)\left|u_{n}\right|^{2}}-\sum_{j=0}^{j_{a}} \frac{p_{0}^{j}\left(\alpha_{0}+\epsilon\right)^{j}\left|u_{n}\right|^{2 j}}{j !}\right) d x \\
& \leq C_{0} \int_{B_{R}}\left(e^{p_{0}\left(\alpha_{0}+\epsilon\right)\left(\frac{4 \pi}{\alpha_{0}}-\delta\right) \frac{\left|u_{n}\right|^{2}}{\left\|u_{n}\right\|^{2}}}-\sum_{j=0}^{j_{a}} \frac{\left(p_{0}\left(\alpha_{0}+\epsilon\right)\left(\frac{4 \pi}{\alpha_{0}}-\delta\right)\right)^{j}}{j !} \frac{\left|u_{n}\right|^{2 j}}{\left\|u_{n}\right\|^{2 j}}\right) d x .
\end{aligned}
$$


Using (5.8) and Theorem 2.1 in the last inequality, we can find some $C>0$ such that

$$
\int_{B_{R}}\left(e^{\left(\alpha_{0}+\epsilon\right)\left|u_{n}\right|^{2}}-\sum_{j=0}^{j_{a}} \frac{\left(\alpha_{0}+\epsilon\right)^{j}\left|u_{n}\right|^{2 j}}{j !}\right)^{p} d x \leq C .
$$

Replacing (5.9) in (5.7), we obtain

$$
\int_{B_{R}} \widetilde{f}\left(x, u_{n}\right) u_{n} d x \leq\left\|u_{n}\right\|_{a^{*}}^{a^{*}}+C\left\|u_{n}\right\|_{p^{\prime}} .
$$

By (5.2), we get

$$
\int_{B_{R}} \tilde{f}\left(x, u_{n}\right) u_{n} d x \rightarrow 0 .
$$

On the other hand,

$$
\int_{\mathbf{R}^{2} \backslash B_{R}} \tilde{f}\left(x, u_{n}\right) u_{n} d x \leq \int_{\mathbf{R}^{2} \backslash B_{R}} V(x) u_{n}^{\mu} d x \leq \int_{\mathbf{R}^{2}} V(x) u_{n}^{\mu} d x
$$

Using Lemma 1.1 and the fact that $u_{n} \rightarrow 0$ in $E$, we can suppose up to a subsequence that

$$
\int_{\mathbf{R}^{2}} V(x) u_{n}^{\mu} d x=\left\|u_{n}\right\|_{L_{V}^{\mu}\left(\mathbf{R}^{2}\right)}^{\mu} \rightarrow 0 .
$$

Thus,

$$
\int_{\mathbf{R}^{2} \backslash B_{R}} \tilde{f}\left(x, u_{n}\right) u_{n} d x \rightarrow 0 .
$$

Combining (5.10) with (5.11), we get

$$
\int_{\mathbf{R}^{2}} \tilde{f}\left(x, u_{n}\right) u_{n} d x \rightarrow 0 .
$$

Using the fact that $\left(\left\|u_{n}\right\|\right)$ is bounded and $\left\|\widetilde{J}^{\prime}\left(u_{n}\right)\right\|_{E^{-}} \rightarrow 0$, we obtain

$$
\left|\widetilde{J}^{\prime}\left(u_{n}\right) u_{n}\right| \leq\left\|\widetilde{J}^{\prime}\left(u_{n}\right)\right\|_{E^{-}}\left\|u_{n}\right\| \rightarrow 0 \text {. }
$$

Since,

$$
\widetilde{J}^{\prime}\left(u_{n}\right) u_{n}=\left\|u_{n}\right\|^{2}-\int_{\mathbf{R}^{2}} \widetilde{f}\left(x, u_{n}\right) u_{n} d x .
$$

By (5.12) and (5.13), we have

$$
\left\|u_{n}\right\|^{2}=\widetilde{J}^{\prime}\left(u_{n}\right) u_{n}+\int_{\mathbf{R}^{2}} \tilde{f}\left(x, u_{n}\right) u_{n} d x \rightarrow 0 .
$$

From (5.4), we have $\left\|u_{n}\right\|^{2} \rightarrow 2 d$. Then, $d=0$ which is a contradiction. Thus, $u$ is a nontrivial critical point of $\widetilde{J}$. 
Lemma 5.2. Let $u$ be the critical point of $\widetilde{J}$ given by Proposition 5.1. Then,

$$
\|u\| \leq \sqrt{\frac{4 \pi}{\alpha_{0}}} .
$$

Proof. Since $\widetilde{J}\left(u_{n}\right) \rightarrow d$, we have

$$
\widetilde{J}\left(u_{n}\right)=\frac{\left\|u_{n}\right\|^{2}}{2}-\int_{\mathbf{R}^{2}} \widetilde{F}\left(x, u_{n}\right) d x=d+o_{n}(1) .
$$

Using the fact that $\widetilde{F}(x, s) \geq 0$ for all $(x, s) \in \mathbf{R}^{2}$ and Remark 4.2, we obtain

$$
\left\|u_{n}\right\|^{2} \leq 2 d+o_{n}(1)<\frac{4 \pi}{\alpha_{0}}+o_{n}(1) .
$$

Since, $u_{n} \rightarrow u$ in $E$, we get

$$
\|u\|^{2} \leq \liminf _{n \rightarrow \infty}\left\|u_{n}\right\|^{2} \leq \frac{4 \pi}{\alpha_{0}}
$$

this complete the proof.

\section{Proof of Theorem 1.3}

Let $0 \neq u \in E$ given by Proposition 5.1. We start showing that

$$
\tilde{f}(x, u(x))=f(u(x)), \quad \text { for all } \quad x \in \mathbf{R}^{2} .
$$

Notice that, by definition $\tilde{f}(x, u(x))=f(u(x))$ for all $|x| \leq R_{0}$. Moreover, if $u(x) \leq 0$ then, $\tilde{f}(x, u(x))=0=f(u(x))$. Thus, we can assume that $u(x)>0$ for all $|x|>R_{0}$. From $\left(H_{4}\right)$, there exist constants $C_{1}=C_{1}(f, \mu, \theta)>0$ and $s_{1}>0$ such that

$$
\frac{f(s)}{s^{\mu-1}} \leq C_{1} s^{\theta} \leq C_{1} s^{\theta} e^{\left(\alpha_{0}+1\right)|s|^{2}}, \quad \text { for all } \quad 0<s<s_{1} .
$$

From $\left(H_{5}\right)$, we have

$$
\lim _{s \rightarrow+\infty} \frac{f(s)}{s^{\mu-1} s^{\theta} e^{\left(\alpha_{0}+1\right)|s|^{2}}} \rightarrow 0 .
$$

Thus, there exist constants $C_{2}=C_{2}\left(f, \mu, \alpha_{0}, \theta\right)>0$ and $s_{2}>0$ such that

$$
\frac{f(s)}{s^{\mu-1}} \leq C_{2} s^{\theta} e^{\left(\alpha_{0}+1\right)|s|^{2}}, \quad \text { for all } \quad s>s_{2}
$$


From the continuity of $f$, there exists a constant $C_{3}=C_{3}\left(f, \mu, \alpha_{0}, \theta\right)>0$ such that

$$
\frac{f(s)}{s^{\mu-1} s^{\theta} e^{\left(\alpha_{0}+1\right)|s|^{2}}} \leq C_{3}, \quad \text { for all } \quad s_{1} \leq s \leq s_{2} .
$$

Combining the estimates (6.2), (6.3) with (6.4), there exists a positive constant $C=C\left(f, \mu, \alpha_{0}, \theta\right)$ such that

$$
\frac{f(s)}{s^{\mu-1}} \leq C s^{\theta} e^{\left(\alpha_{0}+1\right)|s|^{2}}, \quad \text { for all } s>0 .
$$

Since, we suppose that $u(x)>0$ for all $|x|>R_{0}$, we have

$$
\frac{f(u)}{u(x)^{\mu-1}} \leq C u(x)^{\theta} e^{\left(\alpha_{0}+1\right)|u|^{2}}, \quad \text { for all } \quad|x|>R_{0} .
$$

By Lemma 2.1 and the fact that $L_{a} \geq 1$, we have

$$
\frac{f(u)}{u(x)^{\mu-1}} \leq C \frac{\|u\|^{\theta} e^{\left(\alpha_{0}+1\right) \frac{\|u\|^{2}}{|x|^{\frac{2-a}{2}}}}}{|x|^{\left(\frac{2-a}{4}\right) \theta}}, \text { for all }|x|>R_{0} .
$$

Using Lemma 5.2 and the fact that $R_{0} \geq 1$, we get

$$
\frac{f(u)}{u(x)^{\mu-1}} \leq \frac{C(4 \pi)^{\theta / 2} e^{\frac{\left(\alpha_{0}+1\right) 4 \pi}{\alpha_{0}}}}{\alpha_{0}^{\theta / 2}|x|^{\left(\frac{2-a}{4}\right) \theta}}, \text { for all }|x|>R_{0} .
$$

Set

$$
L^{*}:=\frac{C(4 \pi)^{\theta / 2} e^{\frac{\left(\alpha_{0}+1\right) 4 \pi}{\alpha_{0}}}}{\alpha_{0}^{\theta / 2}}
$$

Since $\theta \geq \frac{4 a}{2-a}$, we get

$$
\frac{f(u)}{u(x)^{\mu-1}} \leq \frac{L^{*}}{|x|^{\left(\frac{2-a}{4}\right) \theta}} \leq \frac{L^{*}}{|x|^{a}}, \quad \text { for all } \quad|x|>R_{0} .
$$

Moreover, for $L_{a} \geq L^{*}$, we obtain

$$
\frac{f(u)}{u(x)^{\mu-1}} \leq \frac{L_{a}}{|x|^{a}}, \quad \text { for all } \quad|x|>R_{0} .
$$

From $\left(V_{2}\right)$, we obtain

$$
\frac{f(u)}{u(x)^{\mu-1}} \leq V(x), \quad \text { for all } \quad|x|>R_{0} .
$$


Thus,

$$
\widetilde{f}(x, u(x))=\min \left\{f(u(x)), V(x) u(x)^{\mu-1}\right\}=f(u(x)), \quad \text { for all } \quad|x|>R_{0} .
$$

Hence, (6.1) follows.

Since $u$ is a nontrivial critical point of $\widetilde{J}$, we have

$$
\int_{\mathbf{R}^{2}}(\nabla u \nabla \phi+V(x) u \phi) d x=\int_{\mathbf{R}^{2}} \tilde{f}(x, u) \phi d x, \quad \text { for all } \quad \phi \in E .
$$

Using (6.1), we obtain

$$
\int_{\mathbf{R}^{2}}(\nabla u \nabla \phi+V(x) u \phi) d x=\int_{\mathbf{R}^{2}} f(u) \phi d x, \quad \text { for all } \quad \phi \in E .
$$

That is, equation (1.7) possesses a nontrivial weak solution.

\section{References}

[1] Adimurthi, P. N. Srikanth and S. L. Yadava, Phenomena of critical exponent in $\mathbf{R}^{2}$ Proc. Royal Soc. Edinb. 119A, pp. 19-25, (1991).

[2] Adimurthi and S. L. Yadava, Multiplicity results for semilinear elliptic equations in a bounded domain of $\mathbf{R}^{2}$ involving critical exponents. Ann. Scuola Norm. Sup. Pisa Cl. Sci. (4) 17, pp. 481-504. (1990).

[3] F. S. B. Albuquerque, C. O. Alves and E.S. Medeiros, Nonlinear Schrödinger equation with unbounded or decaying radial potentials involving exponential critical growth in $\mathbf{R}^{2}$. J. Math. Anal. Appl, 409, pp. 1021-1031, (2014).

[4] D. M. Cao, Nontrivial solution of semilinear elliptic equation with critical exponent in $\mathbf{R}^{2}$. Comm. Partial Differential Equations 1, pp. 407435, (1992).

[5] D. G. de Figueiredo, O. H. Miyagaki and B. Ruf, Elliptic equations in $\mathbf{R}^{2}$ with nonlinearities in the critical growth range, Calc. Var. Partial Differential Equations, 3, pp. 139-153, (1995).

[6] C. O. Alves, João Marcos do Ó, and O. H. Miyagaki, On nonlinear perturbations of a periodic elliptic problem in $\mathbf{R}^{2}$ involving critical growth, Nonlinear Analysis, 56, pp. 781-791, (2004). 
[7] de Souza. Manassés and João Marcos do Ó, On a singular and nonhomogeneous $N$-Laplacian equation involving critical growth, J. Math. Anal. Appl., 380, pp. 241-263, (2011).

[8] Manasses de Souza On a singular elliptic problem involving critical growth in $\mathbf{R}^{N}$. Nonlinear Differ. Equ. Appl. 18, pp. 199-215, (2011).

[9] João Marcos do Ó and Bernand Ruf, On a Schrödinger equation with periodic potential and critical growth in $\mathbf{R}^{2}$, Nonlinear differ. equ. appl. 13, pp. 167-192, (2006).

[10] J. M. do Ó, E. S. Medeiros and U. Severo, A nonhomogeneous elliptic problem involving critical growth in dimension two, J. Math. Anal. Appl., 345, pp. 286-304, (2008).

[11] J. M. do Ó, E. S. Medeiros and U. Severo, On a quasilinear nonhomogeneous elliptic equation with critical growth in $\mathbf{R}^{N}$, J. Differential Equations, 246, pp. 1363-1386, (2009).

[12] João Marcos. do Ó, Marco A. S. Souto On a Class of Nonlinear Schrodinger Equations in $\mathbf{R}^{2}$ Involving Critical Growth, Journal of Differential Equations, 174, pp. 289-311, (2001)

[13] J. M. do Ó, N-Laplacian equations in $\mathbf{R}^{N}$ with critical growth, Abstr. Appl. Anal., 2, pp. 301-315, (1997).

[14] O. Kavian, Introduction à la théorie des points critiques et applications aux problèmes elliptiques, Springer-Verlag, Paris, (1993).

[15] J. Moser, A sharp form of an inequality by N. Trudinger, Indiana Univ. Math. J., 20, pp. 1077-1092, (1970/71).

[16] S. Pohožaev, The Sobolev embedding in the special case $p l=n$, Proceedings of the Tech. Sci conference on Adv. Sci. research Mathematics sections 1964-1965, Moscow. Energet. Inst., pp. 158-170, (1965).

[17] Soares, Sérgio H. Monari and Leuyacc, Yony R. Santaria Hamiltonian elliptic systems in dimension two with potentials which can vanish at infinity, Commun. Contemp. Math. 20 (2018), No. 8, 1750053, 37, pp.

[18] J. Su, Z. Q. Wang and M. Willem, Nonlinear Schrödinger equations with unbounded and decaying radial potentials, Commun. Contemp. Math. 9, pp. 571-583, (2007). 
[19] J. Su, Z. Q. Wang and M. Willem, Weighted Sobolev embedding with unbounded and decaying radial potentials, J. Differential Equations, 238, pp. 201-219, (2007).

[20] N. S. Trudinger, On embedding into Orlicz spaces and some applications,J. Math. Mech., 17, pp. 473-483, (1967).

[21] M. Willem, Minimax Theorems, Boston: Birkhäuser, (1996).

\author{
Yony Raúl Santaria Leuyacc \\ Universidade de São Paulo \\ Brasil \\ e-mail : yonyraul@alumni.usp.br
}

